\title{
On the Design of Autonomic, Decentralized VPNs
}

\author{
David Isaac Wolinsky, Kyungyong Lee, P. Oscar Boykin, Renato Figueiredo \\ University of Florida
}

\begin{abstract}
Decentralized and P2P (peer-to-peer) VPNs (virtual private networks) have recently become quite popular for connecting users in small to medium collaborative environments, such as academia, businesses, and homes. In the realm of VPNs, there exist centralized, decentralized, and P2P solutions. Centralized systems require a single entity to provide and manage VPN server(s); decentralized approaches allow more than one entity to share the management responsibility for the VPN infrastructure, while existing P2P approaches rely on a centralized infrastructure but allow users to bypass it to form direct low-latency, highthroughput links between peers. In this paper, we describe a novel VPN architecture that can claim to be both decentralized and P2P, using methods that lower the entry barrier for VPN deployment compared to other VPN approaches. Our solution extends existing work on IP-over-P2P (IPOP) overlay networks to address challenges of configuration, management, bootstrapping, and security. We present the first implementation and analysis of a P2P system secured by DTLS (datagram transport layer security) along with decentralized techniques for revoking user access.
\end{abstract}

\section{INTRODUCTION}

A Virtual Private Network (VPN) provides the illusion of a local area network (LAN) spanning a wide area network (WAN) by creating secure ${ }^{1}$ communication links amongst participants. Common uses of VPNs include secure access to enterprise network resources from remote/insecure locations, connecting distributed resources from multiple sites, and establishing virtual LANs for multiplayer video games and media sharing over the Internet.

The architecture described in this paper addresses usage scenarios where VPNs are desired but complexity in deployment and management limits their applicability. These include collaborative academic environments linking individuals spanning multiple institutions, where coordinated configuration of network infrastructure across different sites is often impractical. Similarly, small/medium business (SMB) environments often desire the ability to securely connect desktops and servers across distributed sites without incurring the complexity or management costs of traditional VPNs. Such a VPN could be used to enable extended families to share media among themselves, such as family videos and pictures, where existing VPNs may be too complicated and where hosting by centralized service may be undesirable for privacy reasons.

The model of a VPN for collaboration considered in this paper is motivated from our Archer [1] project. Archer provides a dynamic and decentralized grid environment for computer architecture researchers to share and access voluntary compute cycles with each other. Use of centralized systems would

\footnotetext{
${ }^{1}$ For the remainder of this paper, unless explicitly stated otherwise, security implies encryption and mutual authentication between peers.
}

limit the scope of Archer and require dedicated administration, whereas existing decentralized solutions require manual configuration of links between peers, which is beyond the scope of Archer's target users. Current P2P virtual network (VN) approaches either lack scalability or proper security components to be considered VPNs.

We began our original foray into user-friendly $\mathrm{VN}$ approaches with IPOP [2]. Previous work on IPOP focused on the routing mechanisms and address allocation with multiple virtual networks (VNs) sharing a single P2P overlay. A shared overlay has significant drawbacks as misconfigured or malicious peers could potentially disable the entire overlay, rendering all VNs useless. Though if security and hence isolation is important, prior to VN deployment, all nodes would need to be configured with a security stack than the $\mathrm{P} 2 \mathrm{P}$ infrastructure prior to deploying the $\mathrm{VN}$ system in order to create a VPN, given the complexity many users would probably reconsider the $\mathrm{P} 2 \mathrm{P}$ approache and use a simple centralized VPN.

To address this challenge, or to make a fully decentralized P2P VPN, in this paper, we extend the IPOP concept to support bootstrapping from public infrastructures and overlays into private and secure P2P overlays whose membership is limited to an individual VPN user base. Our work is based upon Castro et al. [3], suggesting that a single overlay can be used to bootstrap service overlays. We present a practical implementation and evaluation of this concept. We then consider security in the overlay and present the first implementation and evaluation of an overlay with secure communiation both between end points in the P2P overlay (e.g. VPN nodes) as well as between nodes connected by overlay edges. Security requires a means for peer revocation; however, current revocation techniques rely on centralized systems such as certificate revocation lists (CRLs). Our proposed approach allows revocation using scalable techniques provided by the P2P overlay itself. We call the completed system and the interface used to administrate it GroupVPN, a novel decentralized P2P VPN.

The rest of this paper is organized as follows. IPOP along P2P overlays are introduced in Section II. Throughout the paper, there are two techniques used to evaluate our approaches, simulation and real system deployments, these are described in Section III. Section IV describes our techniques that allow users to create their own private overlays from a shared public overlay in spit of NATs. Use of security protocols has been assumed in many $\mathrm{P} 2 \mathrm{P}$ works though without consideration of implementation and overheads, we investigate implementation issues and overheads of security in P2P with emphasis on P2P VPNs in Section V. Without revocation, use of security is limited, in decentralized systems, 
use of centralized revocation methods do not work, we present novel mechanisms for decentralized revocation in Section VI. The complete system, GroupVPN, is presented in Section VII. Section VIII compares and contrasts our work with related work. Section IX concludes the paper.

\section{BACKGROUND}

This section describes the core organization of IPOP, a structured $\mathrm{P} 2 \mathrm{P}$ virtual network, including background on structured overlays, address allocation and discovery, and connectivity.

\section{A. P2P Overlays}

The type of P2P overlay chosen for a VPN has an effect on how easy the VPN is to program, deploy, and secure, on its efficiency, and on its scalability. The two primary infrastructures for P2P overlays are unstructured and structured systems. Unstructured systems use mechanisms such as global knowledge, broadcasts, or stochastic techniques [4] to search the overlay. As the system grows, maintaining and searching typically does not scale. Alternatively, structured approaches provide guaranteed search times typically with a lower bound of $O(\log N)$, where $\mathrm{N}$ is the size of the network. In terms of complexity, for small systems, unstructured systems may be easier to implement, but as the system grows it may become inefficient.

IPOP uses a structured P2P framework named Brunet [5], which is based upon Symphony [6], a one-dimensional ring with a harmonic distribution of shortcuts to far nodes. Structured systems are able to provide bounds on routing and lookup operations by self-organizing into well-defined topologies, such as a one-dimensional ring or a hypercube. Links in the overlay can be made to guarantee efficient lookup and/or routing times (e.g. Brunet automatically creates links between peers that communicate often to achieve efficiency in IP-overP2P communication).

A key component of most structured overlays is support for decentralized storage/retrieval of information such as a distributed hash table (DHT). The DHT builds upon the existence of a P2P address space. All peers in a structured system have a unique, uniformly distributed P2P address. A DHT maps look up values or keys (usually by a hashing function) into the P2P address space. While there are various forms of fault tolerance, a minimalist DHT stores values at the node whose address is closest to the value's key. DHTs can be used to coordinate organization and discovery of resources, making them attractive for self-configuration and organization in decentralized collaborative environments. As explained in the next section, IPOP, uses a DHT to coordinate decentralized organization.

\section{B. Connecting to the VPN}

To connect to IPOP, a peer needs only to connect to an existing Brunet infrastructure. Many IPOP systems can coexist sharing a single overlay. The motivation for doing so is that bootstrapping a P2P system can be challenging, requiring users to have access to public IP addressable nodes or being able to configure a router or firewall to enable inbound connections.
A peer connected to IPOP's P2P infrastructure can take advantage of its support for NAT traversal through hole punching [7]. When performing hole punching, peers first obtain mappings of their private IP address and port to their public IP address and port and then exchange them over a shared medium, in this case the $\mathrm{P} 2 \mathrm{P}$ overlay. The peers attempt to simultaneously form connections with each other, tricking NATs and firewalls into allowing inbound connections, because the NAT believed an outbound flow already exists, thus allowing two machines behind two different NATs direct connectivity. In case peers cannot establish direct connectivity, messages can be relayed through the P2P overlay to each other albeit with added latency and reduced throughput.

This approach enables peers behind NATs and firewalls to seamlessly connect to each other, without requiring peers to host their own bootstrap servers. The requirements for a a bootstrap server include a public IP and the ability to exchange that with users of the system. Though the system should be redundant because if a server in a single bootstrap server system goes offline new nodes will be unable to join the overlay.

\section{Network Configuration}

In the context of VPNs, structured overlays can handle organization of the network space, address allocation and discovery, decentrally through the use of a DHT. Approaches along these lines have been proposed in [8], [9]. Membership in the VPN includes a matching membership in the structured overlay, thus all VPN peers have a P2P address. To address the challenges of having multiple VPNs in the same overlay, each IPOP group has its own namespace, reducing the likelihood of overlap. To enable scalable and decentralized address allocation and discovery, peers store mappings of IP address to P2P address into the DHT, typically of the form hash $($ namespace $+I P)=P 2$ Paddress. Thus a peer attempting to allocate an address will insert this (key, value) pair into the overlay. The first peer to do this will be the owner of the IP address allocation. Therefore the DHT must support atomic writes.

Mechanisms to self-configure the IP address and network parameters of the local system can be provided by DHCP (decentralized host configuration protocol), manually configuring the IP address, or the VPN hooking into O/S APIs. Address discovery is initiated when an outgoing packet for a remote peer arrives at the VPN software. At which point, the VPN will query the DHT with the IP address to obtain the owner's P2P address and forward the packet to the destination. Discussion on both these topics is covered in more depth in our previous work [10].

\section{EXPERIMENTAL ENVIRONMENT}

Throughout this paper, our quantitative evaluation environment uses both real deployments on PlanetLab and simulation. The evaluation requirements dictate the environment used. When the perspective of a single node is useful, PlanetLab provides good results, though when attempting to measure 


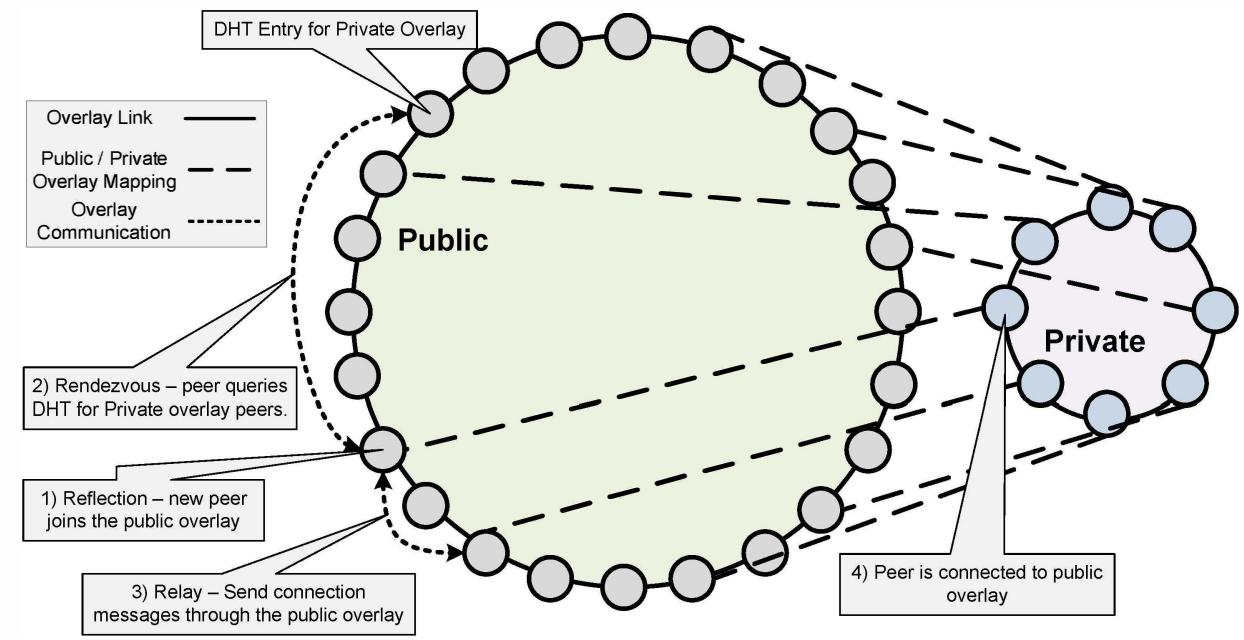

Fig. 1. Bootstrapping a private overlay using Brunet

detailed behavior of the entire system using PlanetLab can result in significant noise.

IPOP uses Brunet as the underlying P2P infrastructure for connectivity. Brunet has been in active development for the past 5 years and is routinely run on PlanetLab [11] for experiments and tests. PlanetLab consists of of nearly 1,000 resources distributed across Earth. In practical applications, though, roughly $40 \%$ of the resources are unavailable at any given time and the remaining behave somewhat unpredictably.

PlanetLab deployment takes approximately 15 minutes for all resources to have Brunet installed and connect to the overlay and then much more time to observe certain behaviors, making regression and verification tests complicated. To address this, we have extended Brunet to support a simulation mode. The simulator inherits all of the Brunet P2P overlay logic but uses simulated virtual time based upon an event-driven scheduler instead of real time. Furthermore, the simulation framework uses a specialized transport layer to avoid the overhead of using TCP or UDP on the host system, both of which are limited resources and can hamper the ability to simulate large systems. The specialized transport uses datagrams to pass messages between nodes, thus from the node's perspective, it is very similar to a UDP transport and can simulate both latency and packet dropping. Latency between all node pairs is set to $100 \mathrm{~ms}$.

Both simulation and real system evaluation provide unique advantages. Simulations allow faster than real time execution of reasonable sized networks (up to a few thousand) using a single resource, while enabling easy debugging. In contrast, deployment on real systems, in particular PlanetLab, presents opportunities to add non-deterministic, dynamic behavior into the system which can be difficult to replicate, such as network glitches and long CPU delays on processing.

\section{TOWARDS PRIVATE OVERlays}

Many users of IPOP begin by using our shared overlay and, once comfortable, move towards hosting their own infrastructure. Some are successful without assistance from us, while a majority are not. Network configuration issues tend to be the most common issue preventing users from hosting their own independent IPOP systems. While users were able to easily join the shared overlay, similar attempts to construct their own were hindered and ultimately only successful after receiving feedback from us.

Bootstrapping a P2P system requires expertise in network administration. To enable users to bootstrap their own private overlays, we previously investigated means by which a public overlay could be used to bootstrap a private overlay. Our approach for bootstrapping private systems requires an overlay to support methods for peers to discover each other, relay messages, and obtain their public address mapping as described in [12]. Examples of other potential bootstrap overlays include popular and well established P2P systems, such as Gnutella, Skype, and Kademlia. Our initial work supports bootstrapping from XMPP (Jabber) systems and our own P2P overlay, Brunet. In this paper, we focus on user perceptions of these technologies with emphasis on bootstrap times and performance overheads, unlike our previous work, which verified its utility for bootstrapping private overlays.

To bootstrap from an existing Brunet overlay, peers first insert their public overlay node address into the key represented by hash (\$PrivateOverlayNamespace) and continue to do so regularly until they disconnect, so as to not let the entry become stale and disappear. Peers attempting to bootstrap into the private overlay can then query this key and obtain a list of public overlay nodes that are currently acting as proxies into the private overlay. By using the public overlay as a transport, similar to UDP or TCP, the private overlay node forms bootstrapping connections via the public overlay. At which point, overlay bootstrapping proceeds as normal. The entire process is represented in Figure 1.

In a small private overlay, there is a possibility that none of the members have a public address, making it difficult to provide overlay based NAT traversal. Rather than having a special case for NAT traversal for private overlays, our model has the private overlay share TCP and UDP sockets with the public overlay. This mechanism, referred to as "pathing", allows multiplexing a single UDP socket and listening TCP socket by many overlays. This is only possible due to the generic transports library of the Brunet P2P overlay, which 
does not differentiate UDP, TCP, or even relayed links. Pathing works as a proxy, intercepting a link creation request from a local entity, mapping that to a path, and then requesting from the remote entity a link for that path. The underlying link is then wrapped by pathing and given to the correct overlay node, resulting in a completely transparent multiplexing of a TCP and UDP sockets, thereby enabling the NAT traversal in one overlay to benefit the other. Once a link has been established, the pathing information is irrelevant, limiting the overhead into the system to a single message exchange during link establishment.

\section{A. Time to Bootstrap a Private Overlay}

This experiment focuses on the overheads in bootstrapping a private overlay using our techniques mentioned in the previous section. The time to bootstrap can be derived analytically by considering the minimum steps for a node to join the public overlay, obtain private overlay peers from the public overlay DHT, and then connect to the private overlay. In Brunet, peers begin by forming leaf or bootstrapping connections and use these to communicate with the neighbor or peer in the P2P network nearest to their P2P address. The process to form a connection can be done in as few as 4 messages and up to 6 , if the peers only know each other's P2P address, which is the case for neighbor connections.

Assuming a peer already has IP address information for another, a connection can be initiated by the peer sending a message to the remote peer expressing the desire for a connection. The remote node responds by either rejecting the request or committing to the connection. In the next exchange, the initiating peer commits to forming the connection and the remote peer acknowledges. The two phase commit process is used to handle the complexity that ensues when multiple simultaneous connection attempts occur in parallel. All these messages take 1 hop, since they are direct links between peers.

When peers only have each other's P2P address and/or the initiating peer is behind a NAT, it may take fifth and sometimes a sixth message. These messages are requests for the remote peer's IP addresses as well as asking the peer to connect with the initiating peer, addressing the case where the remote peer is behind a NAT and cannot handle inbound messages. These messages are routed over the overlay taking $\log (N)$ hops, where $N$ is the network size of the public overlay.

Private overlay bootstrapping follows a similar process, though, first, the peer acquires P2P addresses of other participants through the public DHT, an operation taking $2 * \log (N)$ hops. In the private overlay, the leaf connections do not communicate directly; rather, they use the public overlay, causing some of the 1 hop operations above to take $\log (N)$ hops. Finally, the finding the nearest remote peer in the private overlay takes $\log (N)+\log (n)$, where $n$ is the network size of the private overlay.

Given this model, each operation takes the following hop counts: public overlay bootstrapping $=>8+\log (N)$, DHT operations $=>2 * \log (N)$, and private overlay bootstrapping $=>4+5 * \log (N)+\log (n)$. The cumulative operation takes
$12+8 * \log (N)+\log (n)$ hops. The dominating overhead in bootstrapping the private overlay is the time it takes to perform overlay operations on the public overlay $(\log (N))$. For instance, assuming a network size of 512 public and 8 private, a node should be connected within 87 hops.

To evaluate our implementation for GroupVPN, we use both PlanetLab and the simulator. 100 tests were run for various network sizes. Though due to difficulty in controlling network sizes in PlanetLab, we set each PlanetLab node to randomly decide if it would connect to the private overlay. The network sizes were then used in the simulator and the analytical model. The average public network size for each of these tests was 600. Our results are presented in Figure $2^{2}$.

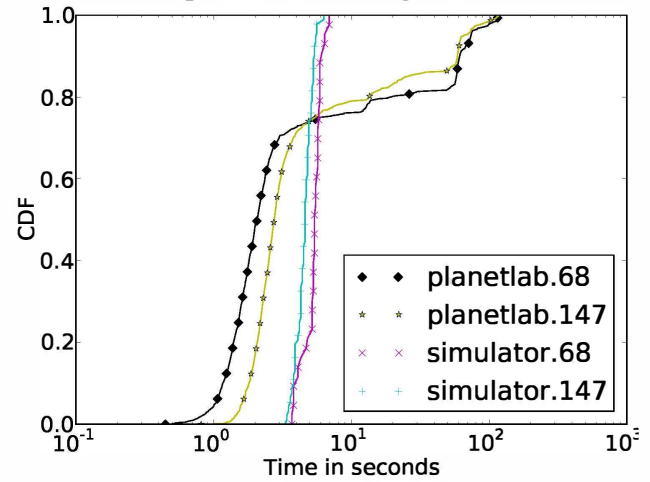

Fig. 2. CDF of the time to bootstrap a private overlay node in a private overlay of the size stated in the legend using a public overlay consisting of 600 nodes. Using a $100 \mathrm{~ms}$ delay like the simulator results in 9.2 and 9.3 seconds for the analytical model for private network sizes of 68 and 147 , respectively.

Based upon the results presented in Figure 2, the bootstrapping time for the implementation performs better than the analytical model, due to the simplicity of the analytical model and the small network sizes. It is of interest that while the simulator results tend to be in a well defined range, the PlanetLab results have a few outliers with long bootstrap times. Some of the expected causes for this are churn in the system and state machine timeouts in Brunet, though we have not considered this in this in much depth in this work.

\section{B. Overhead of Pathing}

Much like the previous experiment, this verifies that the pathing technique has negligible overheads for VPN usage. To determine the overheads, two GroupVPNs are deployed on resources on the same gigabit LAN. To measure latency and throughput, netperf experiments are run for 30 seconds, 5 times each on an unutilized network switch. Other specifications of the machine are ignored as the system without pathing is used as the baseline. The results, Table I, indicate that the use of pathing presents negligible overhead for both throughput and latency, justifying the use of this approach to transparently deal with NAT and firewall traversal.

\footnotetext{
${ }^{2}$ We performed measurements for many more private network sizes, but all the results were so similar that it did not introduce anything of interest and are omitted from our plots to improve clarity.
} 


\begin{tabular}{|c||c|c|}
\hline & Latency (ms) & Throughput (Mbit/s) \\
\hline \hline Standard & .303 & 225.27 \\
\hline Pathing & .308 & 224.36 \\
\hline \multicolumn{3}{|c|}{ TABLE I } \\
PATHING OVERHEADS
\end{tabular}

\section{SECURITY FOR THE OVERLAY AND THE VPN}

Structured overlays are difficult to secure and a private overlay is not secure if it provides no means to limit access to the system. Malicious users can pollute the DHT, send bogus messages, and even prevent the overlay from functioning, rendering the VPN useless. To address this in means that make sense for VPNs and common users, we have employed a public key infrastructure (PKI) to encrypt and authenticate both communication between peers as well as communication across the overlay, called point-to-point (PtP) and end-to-end (EtE) communication, respectively.

Use of a PKI motivates from the ability to authenticate without a third party, ideal for P2P use, unlike a key distribution centers (KDC) used by other VPNs. A PKI can use either preexchange public keys or a certificate authority (CA) to sign public keys, i.e., certificates. Thus peers can exchange keys and certificates without requiring a third-party to be online.

The reasons for securing PtP and EtE are different. Securing PtP communication prevents unauthorized access to the overlay, as peers must authenticate with each other for every link created. Though once authenticated, a peer can perform malicious acts and since the overlay allows for routing over it, the peer can disguise the origination of the malicious acts. By also employing EtE security, the authenticity of messages transferred through an overlay can be verified. Though EtE security by itself, will not prevent unauthorized access into the overlay. By employing both PtP and EtE, overlays can be secured from uninvited guests from the outside and can identify malicious users on the inside. Implementing both leads to important questions: what mechanisms can be used to implement both and what are the effects of both on an overlay and to a VPN on an overlay.

\section{A. Implementing Overlay Security}

There are various types of PtP links; for example, there are TCP and UDP sockets, and relays across nodes and overlays. EtE communication is datagram-oriented in IPOP. Traditional approaches of securing communication such as IPsec are not convenient due to complexity, i.e., operating system specific, portability constraints, and lack of common APIs. Security protocols that rely on reliable connections, such as SSL or TLS are undesirable as well as they would require a userpace implementation of reliable streams (akin to TCP). As such, we have implemented an abstraction akin to a security filter as presented in Figure 3, which enables nearly transparent use of security libraries and protocols. To this date, we have implemented both a DTLS [13] filter using the OpenSSL implementation of DTLS as well as a protocol that reuses cryptographic libraries provided by .NET that behaves similarly to IPsec.
A security filter has two components: the manager, and individual sessions or filters. While the individual sessions could act as filters by themselves, by combining with a manager, they can be configured for a common purpose and security credentials. This approach enables the use of security to be transparent to the other components of the system as the manager handles session establishment, garbage collection of expired sessions, and revocation of peers.

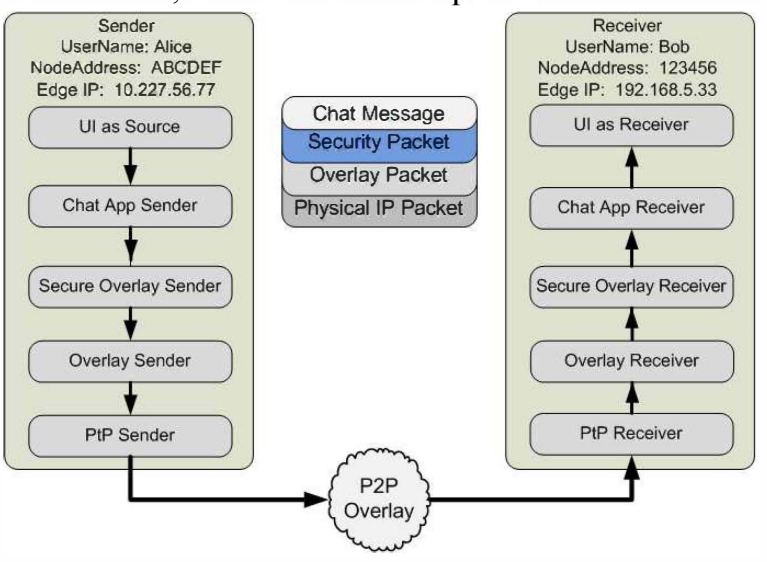

Fig. 3. An example of the abstraction of senders and receivers using a EtE secured chat application. Each receiver and sender use the same abstracted model and thus the chat application requires only high-level changes, such as verifying the certificate used is Alice's and Bob's, to support security.

Certificate embed identity of the owner, thus a signed certificate states that the signer trusts that the identity is accurate. In network systems, the certificate uses the domain name to uniquely identify and limit the use of a certificate. When a CA signs the certificate, by including the domain name, it ensures that users can trust that a certificate is valid, while used to secure traffic to that domain. Communication with another domain using the same certificate will raise a flag and will result in the user not trusting the certificate. In environments with NATs, dynamic IP addresses, or portable devices, typical of $\mathrm{P} 2 \mathrm{P}$ systems, assigning a certificate to a domain name will be a hassle as it constrains mobility and the type of users in the system. Furthermore, most users are unaware of their IP address and changes to it. Instead, a certificate is signed against the user's P2P address and unique user name as delegated by the CA. The purpose of the former is for efficiency of revocation as discussed in Section VI. During the formation of PtP links or while parsing EtE messages, the two nodes discover each other's P2P addresses. If the addresses do not match the address on the verified certificate, the communication need not proceed further.

Prior to trusting the security filter, the core software or the security filter must ensure that the P2P address of the remote entity matches that of the certificate. In our system, we did this by means of a callback, which presents the underlying sending mechanism, EtE or PtP, and the overlay address stored in the certificate. The receiver of the callback can attempt to cast it into known objects. If successful, it will compare the overlay address with the sender type. If unsuccessful, it ignores the request. If any callbacks return that the sender does not match the identifier, the session is immediately closed. Thus 
the security filter need not understand the sending mechanism and the sending mechanism need not understand the security filter.

The last consideration comes in the case of EtE communication that provides an abstraction layer. For example, in the case of VPNs, where a P2P packet contains an IP packet and thus a P2P address maps to a VPN IP address, a malicious peer may establish a trusted link, but then hijack another users IP session. As such, the application must verify that the IP address in the IP packet matches the P2P address of the sender of the P2P packet. In general, an application address should be matched against a $\mathrm{P} 2 \mathrm{P}$ address, consider chat programs, for example.

\section{B. Overheads of Overlay Security}

When applying an additional layer to a P2P system, there are overheads in terms of time to connect with the overlay. Other less obvious effects will be throughput, latency, and processing overheads, assuming that the P2P system will be used over a wide area network, where the latency and throughput limitations between two points will make the overhead of security negligible. Though bootstrapping will be affected due to additional round trip messages used for forming secure connections.

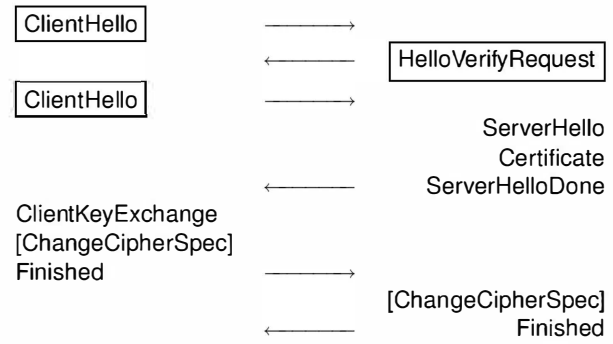

Fig. 4. DTLS handshake

The DTLS handshake as presented in Figure 4, which consists of 6 messages or 3 round trips. PtP security may very well have an effect on the duration of overlay bootstrapping. There even exists a possibility that with more messages during bootstrap, the probability one drops is higher, which could, in turn, also have an effect, though possibly negligible, on time to connect. To evaluate these concerns, we have employed both simulation and real system experiments.

The following experiments use both simulation and PlanetLab deployment to evaluate time to connect a new node to an existing resource. Then another experiment is performed to evaluate how long it takes to bootstrap various sized overlays if all nodes join at the same time. This experiment is only feasible via simulation as attempting to reproduce in a real system is extremely difficult due to how quickly the operations complete.

1) Adding a Single Node: This experiment determines how long it takes a single node to join an existing overlay with and without DTLS security. The experiment is performed using both simulation and PlanetLab. After deploying a set of nodes without security and with security on PlanetLab, the network is crawled to determine the size of the network. In both cases, the overlay maintained an average size of around 600 nodes. At which point, we connected a node 1,000 , each time using a new, randomly generated $\mathrm{P} 2 \mathrm{P}$ address, thus connecting to a different point in the overlay. The experiment concludes as soon as the node has connected to the peers in the P2P overlay immediately before and after it in the P2P address space. In the simulation, a new overlay is created and afterward a new node joins, this is repeated 100 times. The cumulative distribution functions obtained from the different experiments are presented in Figure 5.

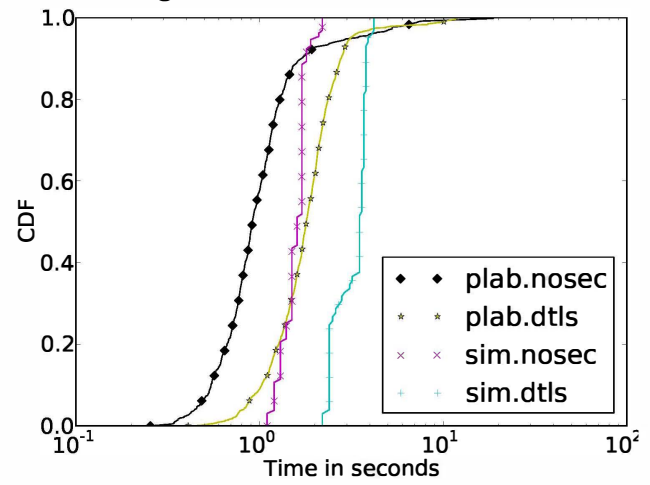

Fig. 5. Time in seconds for a single node to join a secure (dtls) and insecure (nosec) structured overlay, using both PlanetLab (plab) and the Simulator $(\operatorname{sim})$.

2) Bootstrapping an Overlay: The purpose of this experiment is to determine how quickly an overlay using DTLS can bootstrap in comparison to one that does not given that there are no existing participants. Nodes in this evaluation are randomly given information about 5 different nodes in the overlay and then all attempt to connect with each other at the same time. The evaluation completes after the entire overlay has all nodes connected and in their proper position. For each network size, the test is performed 100 times and the average result is presented in Figure 6.

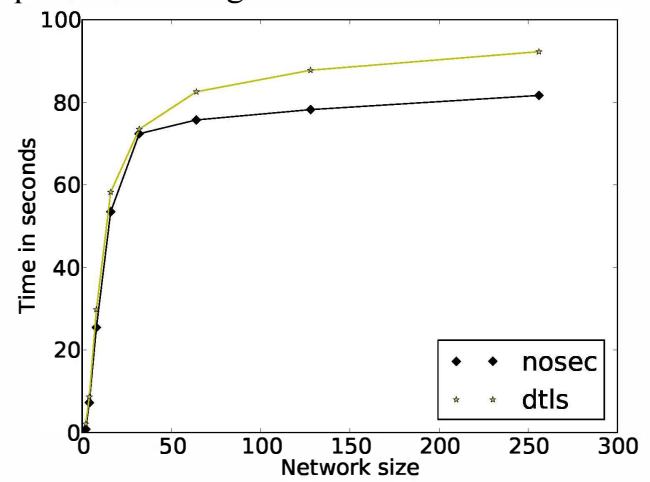

Fig. 6. Time in seconds for a secure (dtls) and insecure (nosec) structured overlay to bootstrap, given that all nodes bootstrap simulataneously.

\section{Discussion}

Both evaluations show that the overhead in using security is practically negligible, when an overlay is small. In the case of adding a single node, it is clear that the simulation and deployment results agree, as the difference between bootstrapping into an overlay with and without security remains nearly 
the same. Clearly this motivates the use of security if time to connect is the most pressing question.

The time to bootstrap a secure overlay was not significantly more than that of an insecure overlay. What we realized is that complex connection handshaking, as implemented in Brunet, seems to dominate connection establishment time. For example, in Brunet, two peers must communicate via the overlay prior to forming a connection, and the system differentiates between bootstrapping connections and overlay connections. Thus even though a peer may have a bootstrapping connection, it will need to go through the entire process to form an overlay connection with a peer. While this may lead to inefficiencies, this simplification keeps the software more maintainable and easier to understand.

\section{HANDLING USER REVOCATION}

Unlike decentralized systems that use shared secrets, in which the creator of the overlay becomes powerless to control malicious users, PKIs enable their creators to effectively remove malicious users. Typical PKIs either use a certificate revocation list (CRL) or online certificate verification protocols such as Online Certificate Status Protocol (OCSP). These approaches are orthogonal to decentralized systems as they require a dedicated service provider. If the service provider is offline, an application can only rely on historical information to make a decision on whether or not to trust a link. In a decentralized system, these features can be enhanced so not to rely on a single provider. In this section, we present two mechanisms of doing so: storing revocations in the DHT and performing overlay broadcast based revocations.

\section{A. DHT Revocation}

A DHT can be used to provide revocation similar to that of OCSP or CRLs. Revocations, a hash of the certificate and a time stamp signed by the $\mathrm{CA}$, are stored are stored in the DHT at the key formed by the hashing of the certificate. In doing so, revocations will be uniformly distributed across the overlay, not relying on any single entity.

The problem with the DHT approach is that it does not provide an event notification for members currently communicating with the peer. While peers could continue to poll the DHT to determine a revocation, doing so is inefficient. Furthermore, a malicious peer, who has a valid but revoked certificate could force every member in the overlay to query the DHT, negatively affecting the DHT nodes storing the revocation.

\section{B. Broadcast Revocation}

Broadcast revocation can be used to address the deficiencies of DHT revocation. As a topic of previous research works [14], [15], structured overlays can be used without additional state to perform efficient broadcasts from any point in the overlay to the entire overlay. The form of broadcast can be used to perform to notify the entire overlay immediately about a new revocation. In these papers, analysis and simulations have shown that the approach can be completed in $O\left(\log ^{2} n\right)$ time.

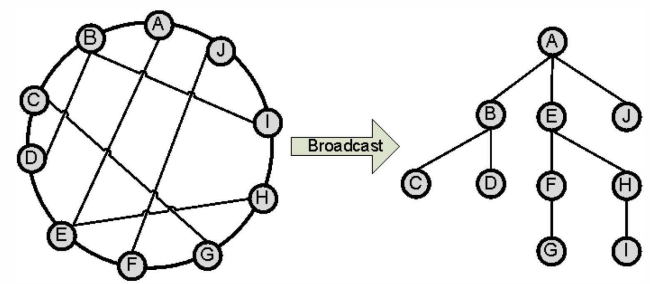

Fig. 7. Broadcast performing a complete overlay broadcast

Our modified algorithm as illustrated in Figure 7 utilizes the organization of a structured system with a circular address space that requires peers be connected to those whose node addresses are the closest to their own, features typical of one-dimensional structured overlays including Chord [16], Pastry [17], and Symphony. Using such an organization, it is possible to do perform a broadcast with no additional state. To perform a broadcast, each node performs the following recursive algorithm:

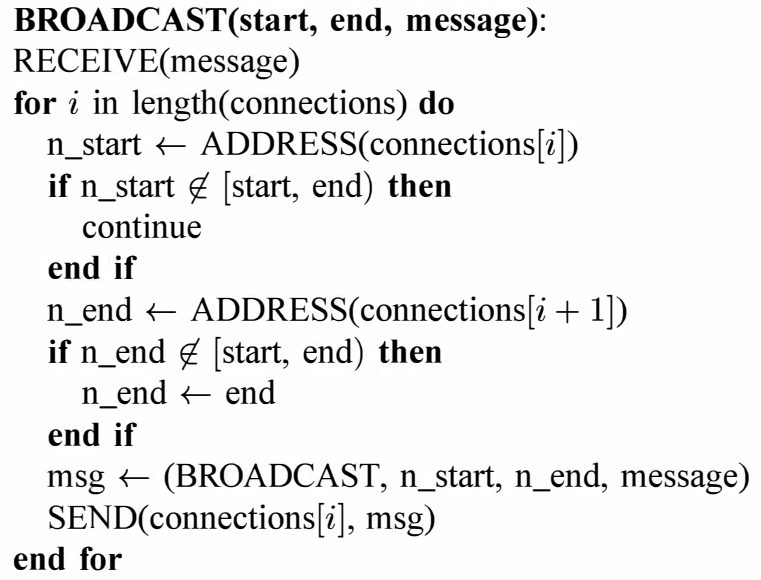

with "connections" as a circular list of connections in nondecreasing order from the perspective of the node performing the current recursive, broadcast step.

In this algorithm, the broadcast initiator uses its own address as the start and end, thus the broadcast will span the entire overlay after completing recursive calls at each connected node. A recursive end, " $n$ _end", must be inside the region between "start" and "end", thus if the connection following the current sending connection, "connections $[i+1]$ ", is not in that region, it will only broadcast up to "end" and not the address specified by that connection. Finally, nodes, who have a connection to the malicious peer, will end the connection prior to accidentally forwarding the message to the peer by receiving and acting upon the revocation prior to forwarding the message. To summarize, the overlay is recursively partitioned amongst the nodes at each hop in the broadcast. By doing so, all nodes receive the broadcast without receiving duplicate broadcast messages.

\section{Evaluation of Broadcast}

We performed an evaluation on the broadcast using the simulation to determine how quickly peers in the overlay would receive the message. The tested network sizes ranged from 2 to 256 in powers of 2 . The tests were evaluations were performed 100 times for each network size. The CDF 
of hops for each node are presented in Figure 8. The results make it quite clear that the broadcast can efficiently distribute a revocation much more quickly than $\log (N)$ time.

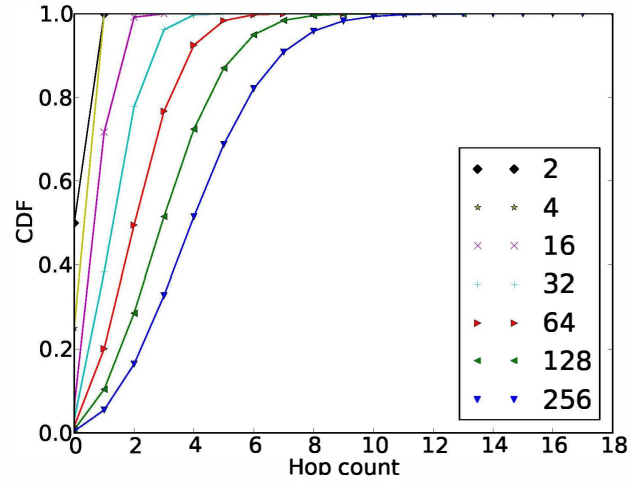

Fig. 8. Overlay broadcast time CDF.

\section{Discussion}

In contrast to the DHT solution, broadcast revocation occur only once and leave no state behind. Thus the broadcast is not a complete solution, as new peers connected to the overlay or those who missed the broadcast message will be unaware of a revocation. Furthermore, if an overlay is shared by many VPNs, it may prevent overlay broadcasting or itself may be inefficient.

The DHT solution by itself may also not sufficient as revocations may be lost over time as the entries must have their leases renewed in the DHT. To address this condition, each peer maintains a local CRL and the owner of the overlay can occasionally send updates to the CRL through an out of band medium, such as e-mail. A better long term solution may be the use of a gossip protocols so that peers can share their lists with each other during bootstrapping phases.

A key assumption in using these is that a Sybil [18], or collusion attack, is difficult in the secured overlay. If a Sybil attack is successful, both a DHT and broadcast revocation may be unsuccessful, though peers could fix this problem by obtaining the CRL out of band. In addition, previous work [19] has described decentralized techniques to limit the probability of such attacks from occurring. In our approach, the use of central authority to review certificate requests can be used to limit a single user from obtaining too many certificates as well as ensuring uniform distribution of that user's P2P addresses, further hampering the likelihood of a Sybil attack. The ability to automate this is left as future work.

One way to mitigate sybil attacks using the broadcast approach is to bundle colluding offenders into a single revocation message. That would prevent those from colluding together to prevent each other's revocations. Furthermore, while not emphasized above, revocation in our system revokes by user name and not individual certificates. Combined these two components limit sybil attacks against broadcast.

\section{ViI. MANAGing AND CONFIGURing THE VPN}

While the PKI model applies to P2P overlays, actual deployment and maintenance of security credentials can be too complex to manage, particularly for non-experts. Most PKIenabled systems require the use of command-line utilities and lack methods for assisting in the deployment of certificates and policing users. Our solution to facilitate use of PKIs for nonexperts is a partially-automated PKI reliant on a group-based Web interface distributable in forms of Joomla add-ons as well as a virtual machine appliance. In this environment, groups can share a common Web site, while each group has their own unique CA. Although this does not preclude other methods of CA interaction, experience has shown that it provides a model that is satisfactory for many use cases.

Group-based Web 2.0 sites enable low overhead configuration of collaborative environments. The roles in a group environment can be divided into administrators and users. Users have the ability to join and create groups; whereas administrators define network parameters, can accept or deny join requests, remove users, and promote other users to administrators. By applying this to a VPN, the group environment provides a simple to use wrapper around PKI, where the administrators of the group act as the CA and the members have the ability to obtain signed certificates.

Elaborating further, when a user joins a group, the administrator can enable automatic signing of certificates or require prior review; and when peers have overstayed their welcome, an administrator can revoke their certificate by removing them from the group. Revocations are handled as described in Section VI. In the context of GroupVPN systems, a user revocation list as opposed to a CRL simplifies revocation, since users and not individual certificates will be revoked.

Registered users who create groups become administrators of their own groups. When a user has been accepted into a group by its administrator, they are able to download VPN configuration data from the Web site. Configuration data is loaded by the GroupVPN during its configuration process to specify IP address range, namespace, and security options. The configuration data also stores a shared secret, which uniquely identifies the user, enabling the Web site to automatically sign the certificate (or enqueue it form manual signing, depending on the group's policy). Certificate requests consist of sending a public key and a shared secret over an HTTPS connection to the web server. Upon receiving the signed certificate, peers are able to join the private overlay and GroupVPN, enabling secure communication amongst the VPN peers. The entire bootstrapping process, including address resolution and communication with a peer, is illustrated in Figure 9.

There are many ways of implementing and hosting the Web site. For example, Google offers free hosting of Python web applications through Google Apps, an option available if the user owns a domain. Alternatively, the user could host the group site on a public virtual network. In this case, peers interacting with the GroupVPN would need to connect with the public virtual network in order to create an account, get the configuration data, and retrieve a signed certificate, at which point they could disconnect from it. This does not preclude the use of other social mediums nor a central site dedicated to the formation of many GroupVPNs. Many GroupVPNs can 


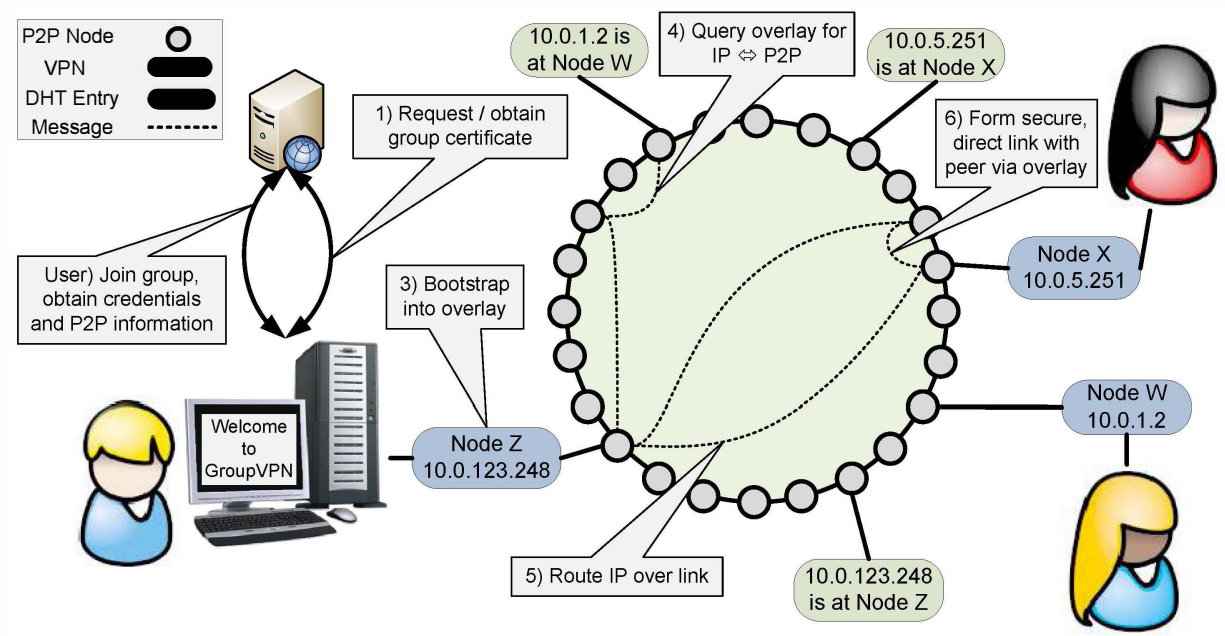

Fig. 9. Process in bootstrapping a new GroupVPN instance.

share a single site, so long as the group members trust the site to host the CA private key.

\section{RELATED WORK}

\section{A. VPNS}

Hamachi [20] is a centralized P2P VPN provider using the web site for authentication, peer discovery, and connection establishment. While the Hamachi protocol claims to support various types of security [21], the implementation appears to only support the key distribution center (KDC) requiring that all peers establish trusted relationship through the central website. The Hamachi approach makes it easy for users to deploy their own services, but places limitations on network size, uses a proprietary security stack, and does not allow independent VPN deployments. In contrast, our approach presents a completely decoupled environment allowing peers to start using our shared system to bootstrap private overlays and migrate away without cost if need be. Furthermore our approach relies only on a central server to obtain the certificate otherwise, it is decentralized. In Hamachi, if the central server goes offline, no new peers can join the VPN.

Campagnol VPN [22] provides similar features to Hamachi: a P2P VPN that relies on a central server for rendezvous or discovery of peers. The key differences between Hamachi and Campagnol is that Campagnol is free and does not provide a service; users msut deploy their own rendezvous service. The authors of Campagnol also state that the current approach limits the total number of peers sharing a VPN to 100 so not to overload the rendezvous service. The current implementation does not support a set of rendezvous nodes, though doing so would make the approach much more like ours. In addition, the system relies on traditional distribution of a CRL to handle revocation.

Tinc [23] is a decentralized VPN requiring users to manually organize an overlay with support for finding optimal paths. In comparison to our approach, Tinc does not automatically handle churn in the VPN. If a node connecting two separate pieces of the VPN overlay goes offline, the VPN will be partitioned until a user manually creates a link connecting the pieces. Furthermore, Tinc does not form direct connections for improved latency and throughput reasons, thus members acting as routes in the overlay incur the price of acting as packet forwarders.

The last VPN, we discuss is the most similar like ours, its called N2N [24]. N2N uses unstructured p2p techniques to form an Ethernet based VPN. While their approach, like ours, has built-in NAT traversal, it requires that users deploy their own bootstrap and limits security to a single pre-shared key for the entire VPN, thus users cannot be revoked. Since N2N provides Ethernet, users must provide their own mechanism for IP address allocation, while discovery utilizes overlay broadcasting. Thus there are concerns that as systems get larger, N2N may not be very efficient.

\section{B. P2P Systems}

BitTorrent [25], a P2P data sharing service, supports stream encryption between peers sharing files. The purpose of BitTorrent security is to obfuscate packets to prevent traffic shaping due to packet sniffing. Thus BitTorrent security uses a weak stream cipher, RC4, and lacks peer authentication as symmetric keys are exchanged through an unauthenticated Diffie-Hellman process.

Skype [26] provides decentralized audio and video communication to over a million concurrent users. While Skype does not provide documentation detailing the security of its system, researchers [27], [28] have discovered that Skype supports both EtE and PtP security. Though similar to Hamachi, Skype uses a KDC and does not let users setup their own systems.

As of December 2009, the FreePastry group released an SSL enabled FreePastry [17]. Though relatively little is published regarding their security implementation, the use of SSL prevents its application for use in the overlay and for overlay links that do not use TCP, such as relays and UDP. Thus their approach is limited to securing environments that are not behind NATs and firewalls that would prevent direct TCP links from forming between peers.

\section{CONCLuSIONS}

This paper overviews the architecture implementation of GroupVPN, a system that is the first to demonstrate the 
practical feasibility of using structured overlays as a basis for easy-to-use, group-oriented, P2P VPNs. Explicitly, we have taken common structured overlays and explored organization, public overlays for connectivity, and private overlays for security and then described our GroupVPN which binds them the components together to create collaborative environments for configuration and management of VPNs. This paper extends upon the IPOP virtual network to support user-friendly approaches for users to create and manage their own virtual private networks. To accomplish this, each IPOP system bootstraps into its own unique, secure P2P overlay. This approach not only enables secure communications in IPOP deployments but also enables for more efficient overlay multicast and broadcast.

The use of service overlays significantly improves performance and maintenance. Peers can easily control membership in the overlay and it presents unique opportunities for decentralized revocation. A DHT approach allows results to be stored on the overlay instead of using centralized CRLs and broadcast to immediately notify active participants of a revocation. Ongoing work include investigating slow bootstrap times and determining security concerns of the decentralized revocation techniques. Furthermore, we plan on investigating the use of overlay broadcasting for IP broadcasting and multicasting, though the current approach places an unfair burden on the first few hops of a broadcast.

Without the functionality of GroupVPN, projects like the Grid Appliances and its flagship application, Archer [1], would be impractical. Archer consists of over 500 resources from 5 different universities, including University of Florida, Florida State University, Northeastern University, University of Minnesota, and University of Texas. In the past year, since Archer came online, over 100 unique users have contributed and taken advantage of the voluntary computing cycles. The use of existing decentralized VPNs in Archer and the Grid Appliance would be severely limiting. In the case of $\mathrm{N} 2 \mathrm{~N}$, at least one peer would have to maintain the bootstrap service and address allocations for the VPN. Tinc would require users to manually configure their networking overlays. While Compagnol and Hamachi require the use of well known centralized peers.

The GroupVPN has been used as the virtual network for the Grid Appliance, enabling the creation of decentralized, collaborative environments for computing grids. Recently, grids at La Jolla Institute for Allergy and Immunology and two in Eastern Europe went live using GroupVPN without receiving any technical support from us. Researchers at Clemson University and Purdue have opted for this approach over centralized VPNs as the basis of their future distributed compute clusters and have actively tested networks of over 700 nodes.

\section{ACKNOWLEDGMENTS}

We thank the anonymous reviewers as well as Pierre St. Juste for their useful comments and feedback. This research is sponsored by the National Science Foundation under grants IIP-0758596 and CNS/CRI collaborative awards 0751112, 0750847, 0750851, 0750852, 0750860, 0750868, 0750884, and 0751091. Any opinions, findings and conclusions or recommendations expressed in this material are those of the authors and do not necessarily reflect the views of the NSF.

\section{REFERENCES}

[1] R. J. Figueiredo, et al., "Archer: A community distributed computing infrastructure for computer architecture research and education," in CollaborateCom, November 2008.

[2] A. Ganguly, A. Agrawal, O. P. Boykin, and R. Figueiredo, "IP over P2P: Enabling self-configuring virtual IP networks for grid computing," in International Parallel and Distributed Processing Symposium, 2006.

[3] M. Castro, P. Druschel, A.-M. Kermarrec, and A. Rowstron, "One ring to rule them all: Service discover and binding in structured peer-to-peer overlay networks," in SIGOPS European Workshop, 2002.

[4] M. Castro, M. Costa, and A. Rowstron, "Debunking some myths about structured and unstructured overlays," in Proceedings of Symposium on Networked Systems Design \& Implementation, 2005.

[5] P. O. Boykin, et al., "A symphony conducted by brunet," http://arxiv. org/abs/0709.4048, 2007.

[6] G. S. Manku, M. Bawa, and P. Raghavan, "Symphony: distributed hashing in a small world," in USITS, 2003.

[7] J. Rosenberg, "Interactive connectivity establishment (ICE): A protocol for network address translator (NAT) traversal for offer/answer protocols," http://tools.ietf.org/html/draft-ietf-mmusic-ice-19, October 2008.

[8] A. Ganguly, D. Wolinsky, P. Boykin, and R. Figueiredo, "Decentralized dynamic host configuration in wide-area overlays of virtual workstations," in International Parallel and Distributed Processing Symposium, March 2007.

[9] I. Stoica, D. Adkins, S. Zhuang, S. Shenker, and S. Surana, "Internet indirection infrastructure," IEEE/ACM Transactions on Networking, 2004.

[10] D. I. Wolinsky, Y. Liu, P. S. Juste, G. Venkatasubramanian, and R. Figueiredo, "On the design of scalable, self-configuring virtual networks," in IEEE/ACM Supercomputing, November 2009.

[11] B. Chun, et al., "Planetlab: an overlay testbed for broad-coverage services," SIGCOMM Comput. Commun. Rev., 2003.

[12] D. I. Wolinsky, P. St. Juste, P. O. Boykin, and R. Figueiredo, “Addressing the P2P bootstrap problem for small overlay networks," in 10th IEEE International Conference on Peer-to-Peer Computing, 2010.

[13] E. Rescorla and N. Modadugu. (2006, April) RFC 4347 datagram transport layer security.

[14] S. El-Ansary, L. Alima, P. Brand, and S. Haridi, "Efficient broadcast in structured p2p networks," in 2nd International Workshop on Peer-toPeer Systems, 2003.

[15] V. Vishnevsky, et al., "Scalable blind search and broadcasting over distributed hash tables," in Computer Communications, vol. 31, no. 2, 2008.

[16] I. Stoica, et al., "Chord: a scalable peer-to-peer lookup protocol for internet applications," vol. 11, no. 1, 2003.

[17] A. Rowstron and P. Druschel, "Pastry: Scalable, decentralized object location and routing for large-scale peer-to-peer systems," in International Conference on Distributed Systems Platforms, November 2001.

[18] J. R. Douceur, "The sybil attack," in IPTPS '01: Revised Papers from the First International Workshop on Peer-to-Peer Systems. Springer-Verlag, 2002, pp. 251-260.

[19] M. Castro, et al., "Security for structured peer-to-peer overlay networks," in Symposium on Operating Systems Design and Implementaion, December 2002 .

[20] LogMeIn. (2009) Hamachi. https://secure.logmein.com/products/ hamachi2/

[21] LogMeIn, Inc. (2009) LogMeIn hamachi2 security.

[22] F. Bondoux, "Campagnol : distributed vpn over udp/dtls," http:// campagnol.sourceforge.net, 2010.

[23] G. Sliepen. (2009, September) tinc. http://www.tinc-vpn.org/.

[24] L. Deri and R. Andrews, "N2N: A layer two peer-to-peer vpn," in In the international conference on Autonomous Infrastructure, Management and Security, 2008

[25] (2007, December) Message stream encryption. http://www.azureuswiki. com/index.php/Message_Stream_Encryption.

[26] S. Limited. Skype. http://www.skype.com.

[27] D. Fabrice. (2005, November) Skype uncovered. http://www.ossir.org/ windows/supports/2005/2005-11-07/EADS-CCR_Fabrice_Skype.pdf.

[28] S. Guha, N. Daswani, and R. Jain, "An experimental study of the skype peer-to-peer voip system," in IPTPS'06, 2006. 